\title{
The Effect of Exercise and Shortwave Diathermy Treatment on the Quality of Life of Patients with Chronic Low Back Pain
}

\author{
Seide Karasel' (1), Sema Öncel² (i), İpek Sönmez ${ }^{3}$ (1) \\ 'Department of Physical Medicine and Rehabilitation, Famagusta State Hospital, Famagusta, Cyprus \\ ${ }^{2}$ Department of Physical Medicine and Rehabilitation, Dokuz Eylül University Hospital, İzmir, Turkey \\ ${ }^{3}$ Department of Pschiatry, Near East University Hospital, Nicosia, Cyprus
}

ORCID iDs of the authors: S.K. 0000-0002-7294-6677; S.Ö. 0000-0002-5083-05I8; i.S.0000-000I-8037-5897.

Cite this article as: Karasal S, Öncel S, Sönmez İ. The Effect of Exercise and Shortwave Diathermy Treatment on the Quality of Life of Patients with Chronic Low Back Pain. Cyprus J Med Sci 2020; 5(3): 205-I0.

\section{BACKGROUND/AIMS}

This study aimed to determine the effects of back school therapy, a home exercise program, and shortwave diathermy treatment on the quality of life of patients with chronic low back pain.

\section{MATERIAL and METHODS}

In this interventional study, we evaluated 90 patients who were admitted to our clinic from 2006 to 2007 and had been followed-up for their low back pain complaints that had been present for at least 6 months. These patients were randomized into 3 groups after being subjected to back school therapy and home exercise programs. Placebo shortwave, continuous shortwave diathermy, and pulsed shortwave diathermy treatments were applied to these three groups, respectively. The Short form 36 (SF-36) was used to evaluate the quality of life of the study groups.

\section{RESULTS}

In this study, statistically significant recovery was achieved in all the three groups in terms of physical function, role limitations due to physical problems, and bodily pain criteria. Those who received the diathermy therapy had higher mean scores in terms of all the three criteria mentioned above.

\section{CONCLUSION}

Significant improvements were achieved within the study groups in terms of all the above-mentioned criteria, except for "role limitations due to emotional problems", as measured by SF-36. However, we could not find any significant difference between the groups in terms of the quality of life.

Keywords: Chronic low back pain, short form health survey, shortwave diathermy

\section{INTRODUCTION}

The Global Burden of Disease studies report that chronic low back pain (CBP) is ranked first among the causes of years lived with disability and sixth among the causes of disability-adjusted years of life (I). The National Institutes of Health Research Task Force defined chronic low back pain as "a back pain problem that has persisted for at least 3 months and has resulted in pain on at least half of the days in the past 6 months" (2). Chronic low back pain is distinguished from acute back pain with regard to the duration and underlying conditions or injuries (3). Lower back pain is a cause of significant disability and severe restriction of routine daily activities. Data from studies demonstrate that more than $84 \%$ of all adults experience low back pain in their lives (4-6). CBP, which is evidently a common disorder, is also associated with physical disability and a reduced quality of life (7).

In chronic low back pain patients, numerous interventional physical modalities have been used in addition to physical treatments. Shortwave diathermy is one of such modalities (4). Shortwave diathermy has been used since 1928 for the treatment of low back pain, since high frequency waves (I0-I00 MHz) cause heat increase in deep tissues (8). Shortwave therapy has been shown to reduce pain and muscle spasm, increase pain threshold, trigger vasodilation, enhance the elasticity of connective tissues, and additionally, it increases joint mobility when applied before exercise therapy $(9,10)$. 
Various studies have demonstrated that exercise and back school programs are effective in patients with $C B P$, and these methods positively contribute to the quality of life and also to the emotional state of patients (II-I3). In a randomized controlled study, the effect of back school therapy on the quality of life was assessed using the SF-36 survey, and an improvement was reported in all the criteria (I3). However, we found only a few studies in the literature that investigated the effects of combined treatment with exercise programs and shortwave diathermy on patients with CBP (I4).

Therefore, this study aimed to determine the effects of back school therapy, home exercise program, and shortwave diathermy treatment on the quality of life of patients with chronic low back pain.

\section{MATERIAL and METHODS}

\section{Study Group}

In this interventional study, we evaluated 90 patients who were admitted to our clinic from 2006 to 2007 and had undergone $X$-ray and MR imaging for their low back pain complaints that had been present for at least 6 months.

Patients aged between 40 and 65 years, suffering from low back pain, which was limited to the lumbar, sacral, or lumbosacral regions, for more than 6 months were included in the study.

Patients with any type of neurological deficit, any type of lumbar or thoracic hernia, cardiovascular disease that would prevent exercise; those who had radicular pain, severe osteoporosis, or osteomalacia; patients with uncontrolled diabetes and hypertension; those with infectious diseases, inflammatory diseases, and a history of malignancy; pregnant patients; and those who had any condition that would prevent the use of the short-term diathermy therapy were excluded from the study. The study was approved by the ethics committee of Dokuz Eylül University, Faculty of Medicine (09/II/2006/247). Patients were informed about the study and a written consent was also obtained.

\section{Treatment}

Age, gender, BMl, occupation, and the duration of the low back pain of all the patients were recorded. Patients were randomized to three groups after being subjected to back school therapy and home exercise programs. In the first group ( $n=30)$, a placebo shortwave therapy was used while the device was switched off. In the second group ( $n=30)$, a continuous shortwave diathermy treatment (Curapuls 419) was applied for 20 minutes every day for 15 days (27.12 MHz frequency and $11.06 \mathrm{~m}$ wavelength, $200 \mathrm{~W}$ ). In the third group $(n=30)$, a pulsed shortwave diathermy treatment (Curapuls 419) was performed for 20 minutes every day for 15 days (27.12 MHz frequency, $11.06 \mathrm{~m}$ wavelength, $200 \mathrm{~W}, 0.3$ msec pause). Randomization and evaluation of the treatment practices were performed by a researcher blinded to the study

\section{Main Points:}

- Continous or pulsed short wave diathermy treatment is effective on the quality of life if patients with chronic low back pain protocol. In the study period, no additional treatment modalities were suggested to the patients.

Short-wave diathermy treatment was applied in the outpatient treatment unit of our clinic 5 days a week for a total of 3 weeks (I5 sessions). The exercise programs of the all patients were demonstrated and performed under the physician's supervision on the first day. From thereon after, all the patients continued their exercise program at home. The patients were advised to perform the exercises in sets of 10, for the three different times in a day. The patients were given an exercise diary and were asked to note the days when they performed the exercises. Patients were re-evaluated at the end of the 3-week treatment period and were asked to attend a final follow-up 3 months later. Therefore, patients were evaluated three times: before the treatment, at the end of treatment, and 3 months after the treatment.

\section{Measurements}

Short form-36 (SF-36) was used to evaluate the quality of life of the patients in the study groups. SF-36 is a questionnaire consisting of questions about general health, physical function, physical role, emotional role, social function, pain, vitality, and mental health that will contribute to the final score, and an additional question regarding the health status, which is not scored. Each question contributes to only one subscale. Possible scores for each subscale range from 0-100. A higher score indicates a higher quality of life (I5).

\section{Statistical Analysis}

Power analysis was performed using the PASS II Home software. The size effect was calculated as 0.414 according to the results of previous studies. Power was adjusted to 0.90 with a type I error of 0.05 . The result showed that at least 27 patients will be required in each group.

All the patients' data were imported into SPSS v2l. Test for normality was done using Shapiro Wilk test. Comparison between the groups was done with one-way Analysis of Variances (ANOVA) or the Kruskal-Wallis test regarding normality for continuous variables and Chi Square test for categorical variables. Evaluation of repeated measurements was done with the two-way repeated measurements ANOVA for normally distributed variables. For non-normally distributed variables, time-bound changes within the groups were evaluated with the Friedman's test, while the differences between repeated measurements in each of the groups were compared with the Kruskal-Wallis test. For pairwise comparison, the Bonferroni correction method was used. A p value $<0.05$ were accepted as statistically significant results.

\section{RESULTS}

A total of 90 patients (17 males and 73 females) were included into this study, and the mean age was $51.36 \pm 6.07$ years. These patients were divided into three groups according to the diathermy treatment (placebo, continuous, pulsed; group l, group 2, group 3; respectively). There was no significant difference between the groups in terms of age, body mass index (BMI), educational status, working status, symptom duration, MRI diagnosis, paracetamol intake, and number of days of exercise. There were significantly more males in group 3 than in the other groups $(p=0.044)$ (Table I). 
TABLE I. Summary of Patients' Characteristics Regarding Treatment Groups

\begin{tabular}{lcccc} 
& Group I & Group 2 & Group 3 & P \\
\hline N & 30 & 30 & 30 & N.A \\
Age & $51.47 \pm 6.50$ & $51.63 \pm 6.26$ & $50.97 \pm 5.59$ & 0.908 \\
Gender (Male) & $3(10.00 \%)^{a}$ & $4(13.33 \%)^{a}$ & $10(33.33 \%)^{b}$ & 0.044 \\
BMI & $25.35 \pm 3.82$ & $25.42 \pm 3.66$ & $25.07 \pm 3.26$ & 0.924 \\
Education Status & & & & \\
Primary & $8(26.67 \%)$ & $6(20.00 \%)$ & $9(30.00 \%)$ & 0.794 \\
Secondary & $6(20.00 \%)$ & $9(30.00 \%)$ & $6(20.00 \%)$ & \\
High school & $10(33.33 \%)$ & $12(40.00 \%)$ & $9(30.00 \%)$ & \\
University & $6(20.00 \%)$ & $3(10.00 \%)$ & $6(20.00 \%)$ & \\
Working Position & & & & \\
Standing & $10(33.33 \%)$ & $10(33.33 \%)$ & $5(16.67 \%)$ & 0.257 \\
Housewife & $16(53.33 \%)$ & $15(50.00 \%)$ & $15(50.00 \%)$ & \\
Sitting & $4(13.33 \%)$ & $5(16.67 \%)$ & $10(33.33 \%)$ & \\
Symptom Duration & & & & \\
(years) & $5(0.5-20)$ & $3.5(1-17)$ & $2.25(0.5-30)$ & 0.185 \\
MRI Diagnosis & & & & \\
Bulging & $5(16.67 \%)$ & $9(30.00 \%)$ & $6(20.00 \%)$ & 0.615 \\
Protrusion & $9(30.00 \%)$ & $9(30.00 \%)$ & $14(46.67 \%)$ & \\
Extrusion & $3(10.00 \%)$ & $2(6.67 \%)$ & $2(6.67 \%)$ & \\
Spinal Stenosis & $1(3.33 \%)$ & $2(6.67 \%)$ & $0(0.00 \%)$ & \\
Degeneration & $12(40.00 \%)$ & $8(26.67 \%)$ & $8(26.67 \%)$ & \\
Paracetamol Intake & $0(0-23)$ & $0(0-30)$ & $0(0-9)$ & 0.294 \\
Exercise (Day) & $89.5(10-90)$ & $75(2-90)$ & $70(12-90)$ & 0.976 \\
Data given as mean \pm standard deviation or median (minimum - maximum) \\
for continuous variables regarding normality and frequency(percentage) for \\
categorical variables \\
Same letters denote lack of significant difference between groups & \\
\hline & & & & \\
\hline
\end{tabular}

Regarding the physical function scores, third month scores were significantly higher than before treatment scores for Group I $(p=0.036)$. There was no significant difference between before and after treatment $(p=0.429)$ and also after treatment and $3^{\text {rd }}$ month scores $(p=0.217)$ for Group I. After treatment and $3^{\text {rd }}$ month scores were significantly higher than the initial scores for Group $2(p<0.001)$, while there was no significant difference between after treatment and third month scores $(p=0.999)$. There was no significant difference between the measurements for Group 3 $(p=0.067)$. In addition, there was no significant difference between the groups in terms of an increase in physical function scores $(p=0.334)$ (Table 2) (Figure I).

Regarding the role limitations due to physical problems scores, third month scores were significantly higher than before treatment scores for Group I ( $p<0.001)$. On the other hand, there was no significant difference between before and after treatment scores $(p=0.526)$ as well as after treatment and $3^{\text {rd }}$ month scores ( $p=0.060)$ for Group I. In Group 2, third month scores were significantly higher than before treatment scores ( $p=0.004)$, while there were no significant differences when before and after treatment scores $(p=0.364)$ and after treatment and third month scores $(p=0.999)$ were compared. For group 3, third month scores were significantly higher than before treatment scores

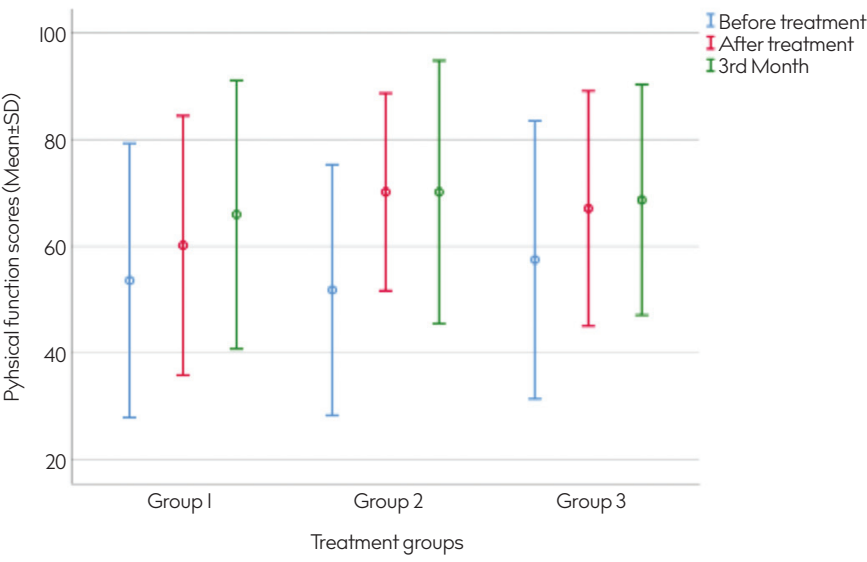

FIGURE I. Isometric flexion strength

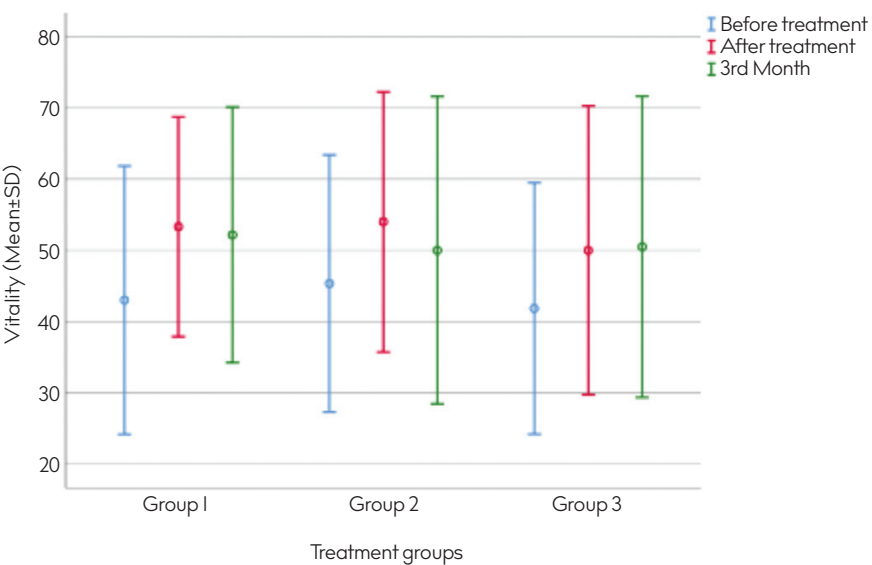

FIGURE 2. Isometric extension strength

$(p=0.006)$, while there was no significant difference between before and after treatment scores $(p=0.320)$ and after treatment and third month scores $(p=0.999)$. There was no significant difference between the groups in terms of an increase in role limitations due to physical problems scores $(p=0.183$ ) (Table 2).

Regarding the bodily pain scores, third month scores were significantly higher than before treatment scores for Group I $(p=0.015)$, while there were no significant differences between before and after treatment scores $(p=0.184)$ and after treatment and third month scores ( $p=0.999)$ for Group I. In Group 2, after treatment and third month scores were significantly higher than before treatment scores $(p<0.001)$. There was no significant difference between after treatment and third month scores $(p=0.999)$. When Group 3 was evaluated, after treatment and third month scores were found to be significantly higher than before treatment scores for Group $3(p<0.001)$. There was no significant difference between after treatment and third month scores $(p=0.364)$. There was also no significant difference between the groups in terms of an increase in bodily pain scores $(p=0.126)$ (Table 2).

Regarding the general health scores, there was no significant difference between the measurements for Group I ( $p=0.198)$. On the other hand, after treatment and third month scores were significantly higher than before treatment scores for Group 2 


\begin{tabular}{|c|c|c|c|c|c|}
\hline & & Group I $(n=30)$ & Group $2(n=30)$ & Group $3(n=30)$ & p (Between Groups) \\
\hline \multirow[t]{3}{*}{ Physical Function } & Before & $53.61 \pm 25.66^{a}$ & $51.83 \pm 23.43^{a}$ & $57.5 \pm 26.06$ & \multirow[t]{3}{*}{0.334} \\
\hline & After & $60.19 \pm 24.29 \mathrm{ab}$ & $70.17 \pm 18.5^{b}$ & $67.09 \pm 22.04$ & \\
\hline & 3rd Month & $65.96 \pm 25.13^{b}$ & $70.17 \pm 24.62^{b}$ & $68.67 \pm 21.57$ & \\
\hline p (Within Groups) & & 0.036 & $<0.001$ & 0.067 & \\
\hline \multirow[t]{3}{*}{ Role Limitations Due to Physical Problems } & Before & $0(0-100)^{a}$ & $25(0-100)^{a}$ & $0(0-100)^{a}$ & \multirow[t]{3}{*}{0.183} \\
\hline & After & $12.5(0-100) \mathrm{ab}$ & $50(0-100) a b$ & $29.17(0-100)^{a b}$ & \\
\hline & 3rd Month & $50(0-100)^{b}$ & $75(0-100)^{b}$ & $50(0-100)^{b}$ & \\
\hline p (Within Groups) & & $<0.001$ & 0.004 & 0.006 & \\
\hline \multirow[t]{3}{*}{ Bodily Pain } & Before & $4 \mathrm{I}(12-100)^{a}$ & $41(10-72)^{a}$ & $41(12-74)^{a}$ & \multirow[t]{3}{*}{0.126} \\
\hline & After & $51(22-84)^{a b}$ & $62(0-84)^{b}$ & $56.5(22-100)^{b}$ & \\
\hline & 3rd Month & $51.5(22-100)^{b}$ & $57(10-100)^{b}$ & $52(10-100)^{b}$ & \\
\hline p (Within Groups) & & 0.015 & $<0.001$ & $<0.001$ & \\
\hline \multirow[t]{3}{*}{ General Health } & Before & $50.43 \pm 19.08$ & $51.37 \pm 17.43^{a}$ & $47.1 \pm \mid 2.1$ & \multirow[t]{3}{*}{0.788} \\
\hline & After & $54.07 \pm 18.95$ & $60.23 \pm 20.09^{b}$ & $52.1 \pm 19.87$ & \\
\hline & 3rd Month & $56.67 \pm 17.82$ & $59.67 \pm 19.21^{b}$ & $52.67 \pm 20.07$ & \\
\hline p (Within Groups) & & 0.198 & 0.011 & 0.283 & \\
\hline \multirow[t]{3}{*}{ Vitality } & Before & $43.00 \pm 18.83^{\circ}$ & $45.33 \pm 18.10^{a}$ & $41.83 \pm 17.64$ & \multirow[t]{3}{*}{0.827} \\
\hline & After & $53.33 \pm 15.44^{b}$ & $54.00 \pm 18.26^{b}$ & $50.00 \pm 20.3$ & \\
\hline & 3rd Month & $52.17 \pm 17.94^{b}$ & $50.00 \pm 21.58^{a b}$ & $50.50 \pm 21.15$ & \\
\hline p (Within Groups) & & 0.010 & 0.041 & 0.059 & \\
\hline \multirow[t]{3}{*}{ Social Function } & Before & $62.5(0-100)$ & $62.5(0-100)$ & $50(12.5-100)^{a}$ & \multirow[t]{3}{*}{0.334} \\
\hline & After & $68.75(12.5-100)$ & $62.5(25-100)$ & $62.5(25-100)^{b}$ & \\
\hline & 3rd Month & $62.5(25-100)$ & $62.5(12.5-100)$ & $62.5(25-100)^{b}$ & \\
\hline p (Within Groups) & & 0.166 & 0.051 & 0.004 & \\
\hline \multirow[t]{3}{*}{ Role Limitations Due to Emotional Problems } & Before & $0(0-100)$ & $66.67(0-100)$ & $0(0-100)$ & \multirow[t]{3}{*}{0.146} \\
\hline & After & $50(0-100)$ & $66.67(0-100)$ & $33.33(0-100)$ & \\
\hline & 3rd Month & $66.67(0-100)$ & $66.67(0-100)$ & $33.33(0-100)$ & \\
\hline$p$ (Within Groups) & & 0.136 & 0.957 & 0.373 & \\
\hline \multirow[t]{3}{*}{ Mental Health } & Before & $57.87 \pm 16.93$ & $59.07 \pm 19.07$ & $51.60 \pm 20.05^{a}$ & \multirow[t]{3}{*}{0.361} \\
\hline & After & $60.80 \pm 15.35$ & $63.07 \pm 19.39$ & $61.47 \pm 20.19^{b}$ & \\
\hline & 3rd Month & $60.53 \pm 17.32$ & $58.00 \pm 17.85$ & $58.40 \pm 18.33^{\mathrm{ab}}$ & \\
\hline $\mathrm{p}$ (Within Groups) & & 0.999 & 0.263 & 0.010 & \\
\hline
\end{tabular}

$(p=0.011)$; however, there was no significant difference between after treatment and third month scores ( $p=0.999)$. In Group 3, there were no significant differences between measurements for Group 3 ( $p=0.283$ ). In addition, there was no significant difference between the groups in terms of the amount of increase in general health scores $(p=0.788)$ (Table 2 ).

Regarding the vitality scores, after treatment and third month scores were significantly higher than before treatment scores for Group I $(p=0.010)$. There was no significant difference between after treatment and third month scores $(p=0.999)$ for this group. In Group 2, after treatment scores were significantly higher than before treatment scores $(p=0.04 \mathrm{I})$, while there was no significant difference between before treatment and third month scores $(p=0.184)$. There was no significant difference between the measurements for Group 3 ( $p=0.059$ ). In addition, there was no significant difference between the groups in terms of the changes in vitality scores $(p=0.827$ ) (Table 2$)$, (Figure 2 ).

Regarding the social function scores, there was no significant difference between measurements for Group I $(p=0.166)$ and Group 2 ( $p=0.05 \mathrm{l}$ ). After treatment and third month scores were significantly higher than before treatment scores for Group 3 $(p=0.004)$. On the other hand, there was no significant difference between after treatment and third month scores $(p=0.999)$. In addition, there was no significant difference between the 
groups in terms of the changes in social function scores $(p=0.334)$ (Table 2).

Regarding the emotional role functioning, no significant differences were found in the comparisons within Group I ( $p=0.136)$, Group 2 ( $p=0.957)$, and Group 3 ( $p=0.373)$, and also in the comparison between the groups $(p=0.146)$.

Regarding the mental health scores, there was no significant difference between the measurements for Group I $(p=0.999)$ and Group 2 ( $p=0.263$ ). For Group 3, after treatment scores were significantly higher than before treatment scores $(p=0.010)$, while there was no significant difference between before treatment and $3^{\text {rd }}$ month scores $(p=0.186)$. Third month scores were lower than after treatment scores, but this difference was not found to be statistically significant for Group $3(p=896)$. In addition, there was no significant differences between the groups in terms of changes in mental health scores $(p=0.361)$ (Table 2 ).

\section{DISCUSSION}

In our study, a statistically significant recovery was achieved in all the three groups in terms of the physical function, role limitations due to physical problems, and bodily pain criteria. All the three groups received back school therapy and home exercise programs, while the first group did not receive the diathermy treatment (placebo). There were significant improvements in all the groups; however, comparisons revealed that there were no significant differences between the groups. Even so, the second and third groups (those that received the diathermy therapy) had higher mean scores in several subscale scores. When within group improvements were evaluated, we found that general health scores were significantly increased in only group 2 (continuous shortwave therapy). There was no significant difference in the vitality criteria in group 3, but there was a significant increase in these criteria in the other groups. On the other hand, there was a significant difference in the social function and mental health scores only in group 3, and no difference was found in the other groups. There was no significant difference in the scores concerning role limitations due to emotional problems in any of the groups.

In a study that evaluated the effects of an exercise program on $\mathrm{CBP}$, improvements in the physical function of the patients were found (16), similar to the findings of our study. However, improvements in role limitations due to physical problems were found in our study whereas, improvements were instead observed in role limitations due to emotional problem in their study (16). Although the authors stated that their study had a significant selection bias that rendered the comparison of the groups quite unfeasible, their findings still showed important benefits with exercise therapy. Furthermore, in a randomized controlled study that evaluated the effect of exercise therapy on the quality of life with the SF-36 questionnaire, significant improvement was shown in all the scores (13). The sample sizes can be a factor that contributes to the differences between the various studies on this topic, but performing exercise therapy under the supervision of a physician or at home (without supervision) may also be a parameter affecting the results. In our study, the exercise programs were demonstrated and performed under a physician's supervision only once. Thereafter, patients were asked to continue their exercise program at home. Although we asked them to keep an exercise diary and to record the time when the exercise was performed, we cannot be absolutely sure that all the exercises were performed in accordance with the demonstration.

In the present study, it was found that there was no difference in terms of the quality of life results in the recipients of the continvous or pulsed shortwave diathermy treatment. In addition, we could not detect a significantly positive effect of the diathermy treatment on the quality of life of patients when compared with placebo. However, in the general health, social function, and mental health scores, no significant change was found in the placebo group, whereas a significant increase was observed in the groups receiving continuous/pulsed diathermy treatment. Several studies have put forth substantial evidence that exercise training can improve the functional ability and quality of life of patients with low back pain $(13,17,18)$. However, there were very few studies that assessed the effect of continuous and pulsed shortwave diathermy on patients with $\operatorname{CBP}(19,20)$. Diathermy treatments are mostly used in the treatment of knee osteoarthritis (2I). In one of these studies, it was reported that shortwave diathermy treatment had no significant effect on the quality of life in knee osteoarthritis (22). Further clinical studies are needed to evaluate the effect of continuous/pulsed shortwave diathermy on the quality of life of patients with chronic low back pain.

The total sample size in the current study can be considered as adequate for an interventional research; however, the fact that the patients were divided into 3 different groups decreased the sample sizes for each group. This may be seen as a limitation, and future studies may benefit from increasing the patient numbers or dividing the patients into two groups. Another limitation in our study is that there was a higher proportion of females than males (approximately $80 \%$ ). Furthermore, approximately half of these females were housewives, which may have been a source of bias. In another study similar to ours, it was reported that the whole study group consisted of women, most of them being housewives, and that this situation may be seen as a handicap because housewives are physically less active, resulting in a bias in terms of the perception of the quality of life among the patients (13). This limitation can be overcome by performing long term studies with larger sample sizes. Finally, pain measurements before and after the interventions and at a follow-up visit would have provided a chance to assess the quality of life results with regard to pain levels; however, pain measurements had not been performed in most of the patients and was therefore lacking from the study, which is also a limitation. An important strength of our study is the fact that, to the best of our knowledge, it is the first study to evaluate the effect of shortwave diathermy treatment on the quality of life of patients with CBP.

In conclusion, the SF-36 questionnaire to evaluate patients with CBP, and all scores -except for role limitation due to emotional problem scores- were significantly increased in each group in our study. However, we could not find any significant difference between the 3 groups in terms of the quality of life. In addition, regarding the general health, social function, and mental health scores, there was no significant change in the placebo group, but significant increases were found in the groups receiving 
continuous or pulsed diathermy treatment. Further clinical studies with larger sample sizes are needed to evaluate the effect of continuous/pulsed short-wave diathermy on the quality of life of patients with chronic low back pain.

Ethics Committee Approval: Ethics committee approval was received for this study from the ethics committee of Dokuz Eylül University, Faculty of Medicine (09/II/2006/247).

Informed Consent: Written informed consent was obtained from patients who participated in this study.

Peer-review: Externally peer-reviewed.

Author contributions: Concept - S.K.; Design - S.K.; Supervision - S.O.; Resource - S.K., S.O.; Materials - S.K.; Data Collection and/or Processing - S.K.; Analysis and/or Interpretation - S.K.; Literature Search - S.K, S.O, I.S.; Writing - S.K, I.S.; Critical Reviews - S.K.

Acknowledgements: Thank for all department of Physical Medicine and Rehabilitation, Dokuz Eylül University, İzmir.

Conflict of Interest: Authors have no conflicts of interest to declare.

Financial Disclosure: The authors declared that this study has received no financial support.

\section{REFERENCES}

I. Vos T, Barber RM, Bell B, Bertozzi-Villa A, Biryukov S, Bolliger I, et al. Global, regional, and national incidence, prevalence, and years lived with disability for $30 \mathrm{I}$ acute and chronic diseases and injuries in 188 countries, 1990-2013: a systematic analysis for the Global Burden of Disease Study 2013. The Lancet 2015; 386(9995): 743-800. [Crossref]

2. Deyo RA, Dworkin SF, Amtmann D, Andersson G, Borenstein D, Carragee $\mathrm{E}$, et al. Focus article: report of the $\mathrm{NIH}$ task force on research standards for chronic low back pain. Eur Spine J 20l4; 23(I0): 2028-45. [Crossref]

3. Chou R, Deyo R, Friedly J, Skelly A, Hashimoto R, Weimer M, et al. Noninvasive treatments for low back pain. 2016.

4. Chou R, Atlas S. Subacute and chronic low back pain: Nonsurgical interventional treatment. UpToDate, Waltham, MA Accessed 20l6; 8(8): 18.

5. Kuijer W, Brouwer S, Preuper HS, Groothoff JW, Geertzen JH, Dijkstra PU. Work status and chronic low back pain: exploring the International Classification of Functioning, Disability and Health. Disabil Rehabil 2006; 28(6): 379-88. [Crossref]

6. Wand BM, O'Connell NE. Chronic non-specific low back pain-subgroups or a single mechanism? BMC Musculoskelet Disord 2008; 9(I): II. [Crossref]

7. Volinn E. The epidemiology of low back pain in the rest of the world: a review of surveys in low-and middle-income countries. Spine 1997; 22(15): 1747-54. [Crossref]
8. Hagen KB, Jamtvedt G, Hilde G, Winnem MF. The updated Cochrane review of bed rest for low back pain and sciatica. Spine 2005; 30(5): 542-6. [Crossref]

9. Low J. Shortwave diathermy, microwave, ultrasound, and interferential therapy. Pain Management in Physical Therapy. 1988.

10. Shields N, Gormley J, O'Hare N. Short-wave diathermy: current clinical and safety practices. Physiother Res Int 2002; 7(4): 191-202. [Crossref]

II. McKillop AB, Carroll LJ, Jones CA, Battié MC. The relation of social support and depression in patients with chronic low back pain. Disabil Rehabil 2017; 39(15): 1482-8. [Crossref]

12. Natour J, Cazotti LdA, Ribeiro LH, Baptista AS, Jones A. Pilates improves pain, function and quality of life in patients with chronic low back pain: a randomized controlled trial. Clin Rehabil 2015; 29(I): 59-68. [Crossref]

13. Tavafian SS, Jamshidi A, Mohammad K, Montazeri A. Low back pain education and short term quality of life: a randomized trial. BMC Musculoskelet Disord 2007; 8(I): 21. [Crossref]

14. Chou R, Huffman LH. Nonpharmacologic therapies for acute and chronic low back pain: a review of the evidence for an American Pain Society/American College of Physicians clinical practice guideline. Ann Intern Med 2007; 147(7): 492-504. [Crossref]

15. Framework IC. The MOS 36-item short-form health survey (SF-36) Med Care 1992; 30(6): 473-83. [Crossref]

16. Claiborne N, Vandenburgh H, Krause TM, Leung P. Measuring quality of life changes in individuals with chronic low back conditions: a back education program evaluation. Eval Program Plann 2002; 25(I): 61-70. [Crossref]

17. Shaughnessy M, Caulfield B. A pilot study to investigate the effect of lumbar stabilisation exercise training on functional ability and quality of life in patients with chronic low back pain. Int J Rehabil Res 2004; 27(4): 297-30l. [Crossref]

18. Van Tulder MW, Koes BW, Bouter LM. Conservative treatment of acute and chronic nonspecific low back pain: a systematic review of randomized controlled trials of the most common interventions. Spine 1997; 22(18): 2128-56. [Crossref]

19. Ahmed MS, Shakoor MA, Khan AA. Evaluation of the effects of shortwave diathermy in patients with chronic low back pain. Bangladesh Med Res Counc Bull 2009; 35(I): 18-20. [Crossref]

20. Gibson T, Harkness J, Blagrave P, Grahame R, Woo P, Hills R. Controlled comparison of short-wave diathermy treatment with osteopathic treatment in non-specific low back pain. The Lancet 1985; 325(8440): 1258-61. [Crossref]

21. Laufer Y, Dar G. Effectiveness of thermal and athermal short-wave diathermy for the management of knee osteoarthritis: a systematic review and meta-analysis. Osteoarthritis Cartilage 2012;20(9):957-66. [Crossref]

22. Akyol Y, Durmus D, Alayli G, Tander B, Bek Y, Canturk F, et al. Does short-wave diathermy increase the effectiveness of isokinetic exercise on pain, function, knee muscle strength, quality of life, and depression in the patients with knee osteoarthritis? A randomized controlled clinical study. Eur J Phys Rehabil Med 20I0; 46(3): 325-36. 